\title{
Enzymfreisetzung und Aktivierung der Kallikrein-Kinin-Systeme bei experimenteller Pankreatitis
}

\author{
Untersuchungen in Pfortaderblut, Pankreaslymphe und Peritonealexsudat
}

\author{
H. Waldner ${ }^{1}$, B. Vollmar ${ }^{2}$, P. Conzen ${ }^{2}$, A. Götz ${ }^{2}$, P. Lehnert ${ }^{3}$, E. Fink ${ }^{4}$, W. Brendel ${ }^{2}$, L. Schweiberer ${ }^{1}$ \\ 1 Chirurgische Klinik und Poliklinik des Klinikums Innenstadt der Ludwig-Maximilians-Universität München \\ 2 Institut für Chirurgische Forschung der Ludwig-Maximilians-Universität München \\ ${ }^{3}$ Medizinische Klinik des Klinikums Innenstadt der Ludwig-Maximilians-Universität München \\ ${ }^{4}$ Abteilung für Klinische Chemie und Klinische Biochemie in der Chirurgischen Klinik und Poliklinik des Klinikums Innenstadt der \\ Ludwig-Maximilians-Universität München
}

Eingegangen: 7. Dezember 1992

Enzyme release and activation of kallikrein-kinin systems in experimental pancreatitis. Measurements in pancreatic blood, lymph and peritoneal exudate

\begin{abstract}
The clinical course of acute pancreatitis is strongly influenced by secondary cardiac, pulmonary and renal damage. The aim of the present study was to gather information about the compartment promoting the systemic damage. Therefore the activity of lipase, phospholipase $\mathrm{A}$ and plasmaprokallikrein and the concentration of tissue kallikrein and kininogen were measured in portal venous blood, pancreatic lymph and peritoneal exudate. Anaesthetized pigs were subjected to fluid resuscitation to keep systemic haemodynamic parameters constant. The pancreas was isolated in situ. The pigs were randomly assigned to a control group $(n=9)$ or one of the two pancreatitis groups ( $n=10$ each). Pancreatitis was induced by i.a. infusion of free fatty acid (FFS) or retrograde infusion of $5 \%$ sodium taurocholate intraductally ( $\mathrm{NaT})$. In both pancreatitis groups the activity of lipase and phospholipase A increased. The most pronounced changes were seen in the peritoneal exsudate (phospholipase A activity 40 min after induction: control $10.0 \mathrm{U} / 1$, NaT $72.2 \mathrm{U} / 1$ ). In both pancreatitis groups there was evidence for activation of the tissue kallikreinkinin system in the form of an increase in the kallikrein concentration and a decrease in the kininogen concentration. Again the changes were most pronounced in the peritoneal exsudate (tissue kallikrein $40 \mathrm{~min}$ after induction: control $14.7 \mathrm{ng} / \mathrm{ml}, \mathrm{NaT} 452 \mathrm{ng} / \mathrm{ml}$ ).
\end{abstract}

Key words: Pancreatitis - Phospholipase - Kallikrein Lymph - Peritoneal exsudate

Zusammenfassung. Das klinische Bild der akuten Pankreatitis wird entscheidend durch die sekundäre Schädigung von Herz-Kreislauf-System, Lunge und Niere bestimmt.

Korrespondenz an: Prof. Dr. H. Waldner, Chirurgische Klinik Innenstadt, Nussbaumstraße 20, W-8000 München 2
Ziel der vorliegenden Untersuchung war es, durch Messungen in venösem Pankreasblut, Pankreaslymphe und Peritonealexsudat die Kompartimente $\mathrm{zu}$ bestimmen, über die die systemischen Schädigungen vermittelt werden. An anästhesierten Schweinen wurden die systemischen, hämodynamischen Parameter durch gesteuerte Volumentherapie konstant gehalten. Die Schweine wurden randomisiert der Kontrollgruppe $(n=9)$ oder einer der Pankreatitisgruppen zugeteilt (jeweils $n=10$ ). Die Pankreatitis wurde durch Infusion von freier Fettsäure in die Pankreasarterien (FFS) oder durch Infusion einer $5 \%$ igen Natrium-Taurocholat-Lösung retrograd in den Pankreasgang (NaT) ausgelöst. Nach Isolation des Pankreas wurde venöses Pankreasblut, Pankreaslymphe und Peritonealexsudat gewonnen und die Aktivität von Lipase, Phospholipase A und Plasmaprokallikrein sowie die Konzentration von Organkallikrein und Kininogen bestimmt. In beiden Pankreatitismodellen fand sich ein Anstieg der Enzymaktivitäten. Die höchsten Aktivitäten fanden sich im Peritonealexsudat (Phospholipase A nach 40 min: Kontrolle 10,0 U/1, NaT 72,2 U/1). In beiden Pankreatitismodellen fanden sich außerdem Hinweise für eine Aktivierung des Organkallikrein-Kinin-Systems durch den Anstieg der Organkallikreinkonzentration und den Abfall der Gesamtkininogenkonzentration. Die stärksten Veränderungen fanden sich wieder im Peritonealexsudat (Organkallikrein nach $40 \mathrm{~min}$ : Kontrolle $14,7 \mathrm{ng} / \mathrm{ml}, \mathrm{NaT} 452 \mathrm{ng} / \mathrm{ml})$.

Nach einer Initialphase mit abdominellen Beschwerden ist der Verlauf der schweren Pankreatitis durch das Versagen lebensnotwendiger Organe wie Herz, Kreislauf [4], Lunge [5] und Niere gekennzeichnet. Dieses Organversagen ist für die hohe Letalität der schweren Pankreatitis verantwortlich. Primäre Ursache für diese Organversagen soll die Aktivierung und Freisetzung von Pankreasenzymen, insbesondere von Proteinasen und Phospholi- 
pase, toxischen Substanzen und vasoaktiven Mediatoren sein [6].

Ziel der vorliegenden Untersuchung war es, bei der akuten Pankreatitis zum einen die initiale Aktivierung von Enzymen und Freisetzung von Mediatoren im Pankreas und seiner unmittelbaren Umgebung nachzuweisen und zum anderen deren Freisetzung in die Kompartimente venöses Pankreasblut, Pankreaslymphe und Peritonealexsudat selektiv zu erfassen.

Über die Enzym- und Mediatorenspiegel bei akuter Pankreatitis liegen für peripheres Blut diverse Untersuchungen vor $[8,11,15]$. Untersuchungen im peripheren Blut zur Aufdeckung pathophysiologischer Veränderungen sind in der Frühphase der Pankreatitis nur bedingt geeignet, da infolge der hohen Proteinasehemmaktivität im Blut und der hohen Clearancekapazität des retikuloendothelialen Systems diskrete Veränderungen damit nicht sicher erfaßt werden können [3].

Die Bedeutung verschiedener Kompartimente wie venöses Pankreasblut, Pankreaslymphe und Peritonealexsudat für die Vermittlung systemischer Wirkungen, die letztendlich bei der akuten Pankreatitis zum Multiorganversagen führen, ist noch nicht geklärt. Als ein erster Schritt dazu wurde in der vorliegenden Untersuchung die Freisetzung der Pankreasenzyme Lipase und Phospholipase sowie die Konzentrationsänderungen von Komponenten der Kallikrein-Kinin-Systeme in den verschiedenen Kompartimenten quantitativ bestimmt. Durch die Bestimmung von Kallikrein und Kininogen sollten Hinweise für eine Freisetzung von Kinin, einem vasoaktiven Mediator, erhalten werden. Um dabei eine möglichst große Aussagekraft zu erreichen, wurden 2 pathogenetisch unterschiedliche Pankreatitismodelle verwendet, nämlich

- die retrograde Injektion von Gallensäure in den Pankreasgang und

- die intraarterielle Infusion von Ölsäure in das Pankreas.

\section{Methoden und Material}

Nach Genehmigung durch die zuständigen Behörden wurden die Untersuchungen an Schweinen mit einem mittleren Körpergewicht von $30 \mathrm{~kg}$ durchgeführt.

Die Narkoseeinleitung erfolgte mit Ketamin $(8 \mathrm{mg} / \mathrm{kg} \mathrm{KG})$, Fluonitrazol $(0,13 \mathrm{mg} / \mathrm{kg} \mathrm{KG})$ und Atropinsulfat $(1,7 \mathrm{mg} / \mathrm{kg} \mathrm{KG})$. Nach Tracheotomie wurden die Tiere beatmet, die Narkose mit Sauerstoff, Lachgas und Enflurane (0,5-1\%) fortgeführt. Die Infusionstherapie mit physiologischer Kochsalzlösung wurde so gesteuert, daß der pulmonal-kapilläre Verschlußdruck und der arterielle Druck während des Versuchsablaufes konstant blieb. Zur Gewinnung von portalvenösem Blut, Lymphe und Exsudat wurde das Pankreas nach folgendem Vorgehen in situ isoliert: es wurden eine Splenektomie und eine Gastrektomie durchgeführt, außerdem wurden Dünn- und Dickdarm entfernt. Das Modell ist an anderer Stelle detailliert beschrieben [24]. Für die portalvenösen Blutentnahmen wurde die Pfortader, die nach oben genannter Präparation nur Pankreasblut führt, über die V. mesenterica superior kanüliert. Um die Lymphe von Zwerchfell, Milz und linksseitigem Pankreas kontinuierlich ableiten zu können, wurde der linke obere Lymphhauptstamm in Höhe der Nierenvene dargestellt und kanüliert [14]. Peritonealexsudat wurde aus der Bauchhöhle kontinuierlich abgesaugt.

\section{Biochemische Untersuchungen}

Die Bestimmung des Gesamteiweißes in Pfortaderserum, Lymphe und Aszites erfolgte mit der Biuret-Methode. Die Aktivität der Enzyme Lipase und Phospholipase A wurde photometrisch bestimmt. Die Proben wurden sofort nach der Entnahme bei $3000 \mathrm{~g}$ zentrifugiert und bei $-20^{\circ} \mathrm{C}$ eingefroren. Für die Messungen wurden sie aufgetaut und sofort bearbeitet. Die Lipase wurde in Pfortaderserum, Lymphe und Peritonealexsudat bestimmt [25]. Die Messungen der Phospholipaseaktivität erfolgte in Pfortaderserum, Lymphe und Peritonealexsudat [9]. Zur Beurteilung der Aktivität der Kallikrein-Kinin-Systeme wurden die Konzentrationen des Plasmaprokallikreins, des Gewebekallikreins und des Gesamtkininogens bestimmt. Nach Abnahme wurden die Proben sofort bei $3000 \mathrm{~g}$ für $10 \mathrm{~min}$ zentrifugiert und bei $-70^{\circ} \mathrm{C}$ eingefroren. Das Plasmaprokallikrein wurde als librierbares Plasmakallikrein gemessen. Die Bestimmung erfolgte auf photometrischer Basis mit einem Farbstofftest von Kabi-Vitrum. Die Messungen wurden im Pfortaderplasma durchgeführt. Gewebekallikrein wurde mit einem spezifischen Radioimmunoassay bestimmt. Die Kininogenkonzentration wurde durch Bestimmung des aus dem Kininogen freisetzbaren Kinins [18] ermittelt. Die Messungen erfolgten in heparinisiertem Pfortaderplasma, heparinisierter Lymphe und heparinisiertem Peritonealexsudat.

\section{Versuchsablauf}

Die Tiere wurden randomisiert einer der Versuchsgruppen zugeteilt, der Beobachtungszeitraum betrug $6 \mathrm{~h}$. Die chirurgische Präparation und das Versuchsprotokoll war in allen Versuchsgruppen gleich.

Nach Erreichen eines Steadystate erfolgten die basalen Messungen. Proben für die Laboruntersuchungen wurden $\mathrm{zu}$ folgenden 5 Zeitpunkten abgenommen: am Ende des Steadystate, nach 40 min, $2 \mathrm{~h}, 4 \mathrm{~h}$ und $6 \mathrm{~h}$.

Am Ende des Steadystates erfolgte die Abnahme von Pfortaderblut und peripankreatischer Lymphe, zu den späteren Zeitpunkten zusätzlich auch von Peritonealexsudat.

Es wurden 3 Versuchsgruppen gebildet:

- Kontrolle $(n=9)$,

- Freie-Fettsäure-Pankreatitis (FFS) $(n=10)$. Die FFS-Pankreatitis wurde durch Infusion von $0,1 \mathrm{ml} / \mathrm{kg} \mathrm{KG}$ reiner Ölsäure (Oleic acid, Sigma) in den Truncus coeliacus über einen Zeitraum von $15 \mathrm{~min}$ induziert.

- Natrium-Taurocholat-Pankreatitis (NaT) $(n=10)$. Die NaTPankreatitis wurde durch Infusion von $1 \mathrm{ml} / \mathrm{kg} \mathrm{KG}$ einer $5 \%$ igen Natrium-Taurocholat-Lösung (Taurocholic acid, Sigma T 0750) unter einem Druck von $20 \mathrm{~cm}$ Wassersäule in den Ductus pancreaticus ausgelöst.

\section{Statistik}

In den Kurven und Tabellen sind jeweils die Mittelwerte und der Standardfehler der Mittelwerte (SEM) angegeben.

Die anaytische Statistik erfolgte für den Innergruppenvergleich mit Rangvarianzanalyse nach Friedman und für den Zwischengruppenvergleich nach der Kruskal-Wallis-Analyse, da eine Normverteilung der Werte nicht angenommen werden konnte. Es handelte sich in beiden Tests um bindungskorrigierte Einzelvergleiche. Im Innergruppenvergleich wurde der Ausgangswert mit dem 40-min-Wert und dem 6 -h-Wert verglichen. Das Signifikanzniveau betrug $<0,05$.

\section{Ergebnisse}

\section{Histologie}

Schon makroskopisch war an den Pankreaspräparaten des Ölsäuremodells ein Ödem zu erkennen. Histologische 
Schnitte des Pankreas zeigten eine Verbreiterung der Interlobärsepten als Zeichen des Ödems. Außerdem waren kleine Nekroseherde im exokrinen Parenchym nachzuweisen, die aber jeweils nur Teile eines Läppchens betrafen. In den Gefäßen dieser Bereiche fanden sich Fibrinthromben.

Durch die Natrium-Taurocholat-Infusion kam es makroskopisch zu fokalen, überwiegend im Kopfbereich lokalisierten hämorrhagischen Pankreasnekrosen. Histologisch fanden sich Nekrosebereiche neben vitalem Parenchym. Die Nekrosebereiche waren v.a. um das Pankreasgangsystem lokalisiert. In den Randbereichen der Nekrosen waren vermehrt Entzündungszellen nachweisbar.

\section{Biochemische Untersuchungen}

Gesamteiweiß. Die Gesamteiweißkonzentration fiel in Serum und Lymphe in allen 3 Versuchsgruppen über die 6stündige Versuchsdauer tendenziell ab; so lagen die Serumwerte in der Kontrollgruppe initial bei 3,4 $( \pm 0,26)$ $\mathrm{g} / \mathrm{dl}$ und nach $6 \mathrm{~h}$ bei $2,0( \pm 0,88) \mathrm{g} / \mathrm{dl}$. Die Gesamteiweißkonzentrationen im Peritonealexsudat zeigten hingegen keine Änderung über die Versuchsdauer; die Werte in der Natrium-Taurocholat-Gruppe waren bei der ersten Messung bei $1,2( \pm 0,23) \mathrm{g} / \mathrm{dl}$ nach $6 \mathrm{~h}$ bei 1,1 $( \pm 0,99) \mathrm{g} / \mathrm{dl}$.

Pankreasenzyme. Die Lipaseaktivität stieg bei beiden Pankreatitisgruppen im Vergleich zur Kontrollgruppe in Serum, Lymphe und im Peritonealexsudat (Abb. 1) signifikant an. In der NaT-Gruppe fand sich im Serum bereits nach $40 \mathrm{~min}$ ein signifikanter Anstieg von $54,4( \pm 5,33)$ $\mathrm{U} / 1$ auf $142( \pm 20,3) \mathrm{U} / \mathrm{l}$. Im Peritonealexsudat war die Lipaseaktivität nach $6 \mathrm{~h}$ bei der FFS-Pankreatitisgruppe um das Doppelte, bei der NaT-Pankreatitis um das 3 fache gegenüber der Kontrolle erhöht (Abb. 1). Unterschiedlich war die Kinetik des Aktivitätsanstiegs in den Pankreatitisgruppen. In der FFS-Gruppe kam es zu einem kontinuierlichen Anstieg der Aktivität in allen Kompartimenten bis zum Versuchsende. Bei der NaTPankreatitis hingegen fand sich ein rascher Anstieg in den Anfangsphasen mit den höchsten Werten innerhalb von $2 \mathrm{~h}$.

In der Kontrollgruppe kam es zu keiner Änderung der Phospholipaseaktivität in Serum, Lymphe und Peritonealexsudat (Abb. 2), sie lag über den gesamten Versuchszeitraum im Normbereich unter $10 \mathrm{U} / 1$. In beiden Pankreatitisgruppen blieben die Phospholipaseaktivitäten in Serum und Lymphe unverändert; die Werte lagen im Normbereich. Im Peritonealexsudat wurde bei der FFS-Pankreatitis ein kontinuierlicher Anstieg der Phospholipaseaktivität beobachtet (Abb. 2). Bei der NaTPankreatitis fand sich bereits innerhalb von $40 \mathrm{~min}$ der PLA-Aktivität im Peritonealexsudat ein signifikanter Anstieg um das 7 fache im Vergleich zur Kontrollgruppe (Abb. 2). Die PLA-Aktivität blieb dann über die ganze Beobachtungsphase erhöht und lag nach $6 \mathrm{~h}$ bei einem Mittelwert von $39( \pm 12)$ U/l. Die PLA-A waren in jeder Gruppe im Peritonealexsudat höher als in der Lymphe.

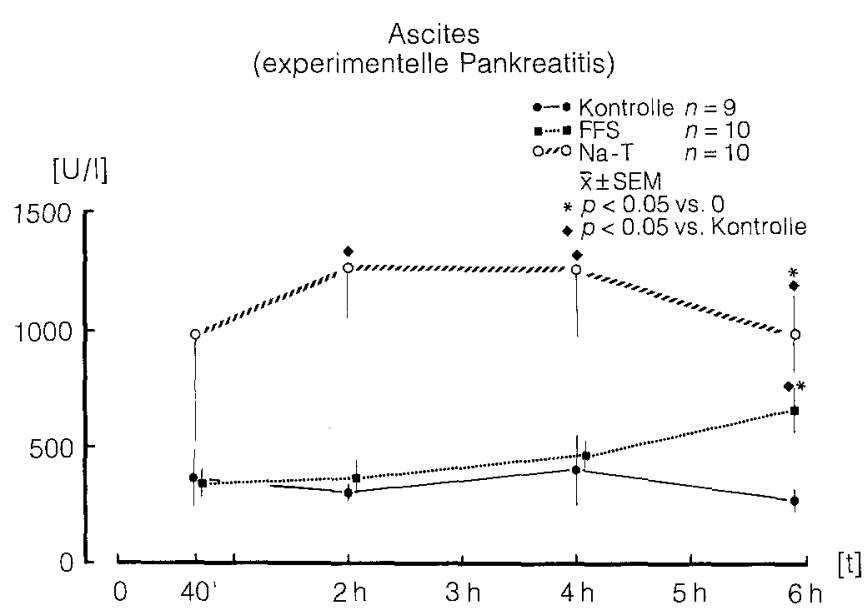

Abb. 1. Dargestellt sind die Mittelwerte ( \pm SEM) der Lipaseaktivität in der Kontrollgruppe und in den beiden Pankreatitisgruppen (FFS und $\mathrm{NaT}$ ) im Peritonealexsudat. Die Werte basal und während der 6 stündigen Beobachtungsphase sind in $U / 1$ angegeben. In der Kontrollgruppe blieb die Lipaseaktivität nahezu konstant bei $300 \mathrm{U} / 1$. In der FFS-Gruppe stieg sie signifikant von 375 auf $640 \mathrm{U} / 1$ an. In der NaT-Gruppe war schon der 40-min-Wert mit 983,6 U/1 stark erhöht; nach $2 \mathrm{~h}$ lagen alle Werte signifikant über denen der Kontrollgruppe

Ascites

(experimentelle Pankreatitis)

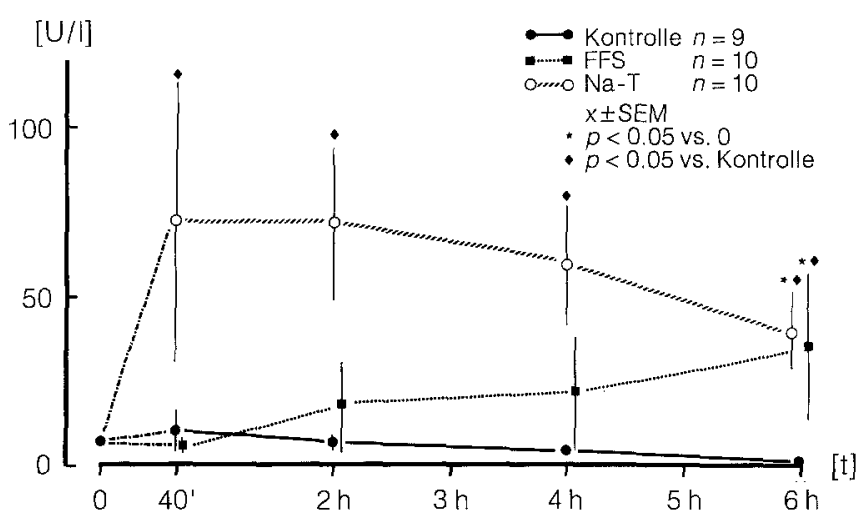

Abb. 2. Dargestellt sind die Mittelwerte ( \pm SEM) der Phospholipase-A-Aktivität in der Kontrollgruppe und in den beiden Pankreatitisgruppen (FFS und NaT) im Peritonealexsudat. Die Werte basal und während der 6stündigen Beobachtungsphase sind in U/1 angegeben. In der Kontrollgruppe lag die Phospholipaseaktivität unter $10 \mathrm{U} / 1$. In der FFS-Gruppe fand sich ein kontinuierlicher Anstieg von 6,5 auf 37,5 U/1. Dieser Anstieg war noch $6 \mathrm{~h}$ signifikant gegenüber dem Ausgangswert und der Kontrollgruppe. Bei der NaTGruppe war schon der 40-min-Wert mit 72 U/1 signifikant gegenüber der Kontrollgruppe erhöht

Kallikrein-Kinin-Systeme. Die Mittelwerte der generierbaren Plasma-Kallikrein-Aktivität im Pfortaderplasma, die der Prokallikreinkonzentration entsprechen, lagen in den Versuchsgruppen basal zwischen $1842( \pm 193) \mathrm{U} / 1$ und $2051( \pm 215) \mathrm{U} / \mathrm{l}$. In der Kontrollgruppe und in der NaT-Gruppe fiel die Konzentration innerhalb von $6 \mathrm{~h}$ leicht $\mathrm{ab}$. In der FFS-Gruppe war dagegen bereits der 40-min-Wert um $41 \%$ erniedrigt und fiel dann noch weiter $\mathrm{ab}$.

Die Konzentration an Gewebekallikrein in der Kontrollgruppe blieb in Pfortaderplasma und peripankreati- 
Ascites

(experimentelle Pankreatitis)

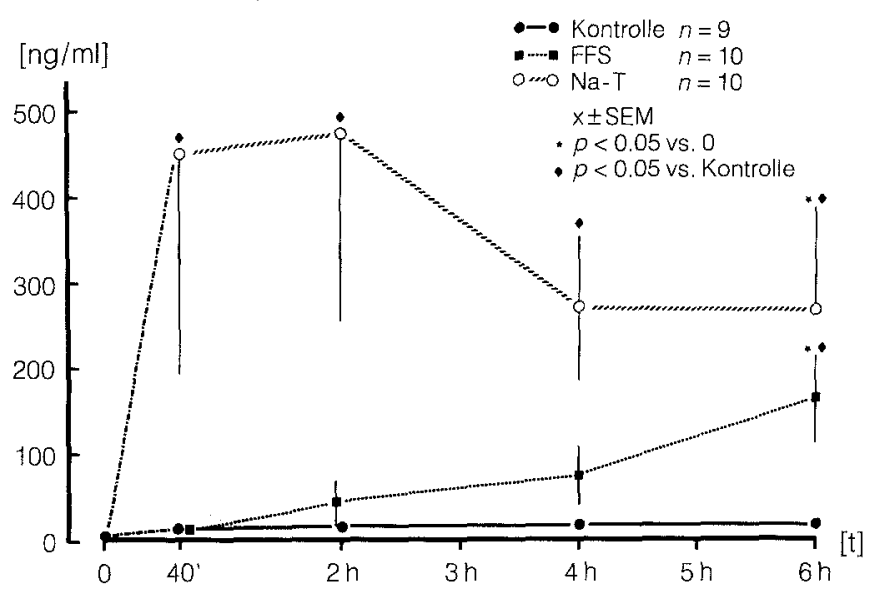

Abb. 3. Dargestellt sind die Mittelwerte ( \pm SEM) der Organkallikreinaktivität in der Kontrollgruppe und in den beiden Pankreatitisgruppen (FFS und NaT) im Peritonealexsudat. Die Werte basal und während der 6 stündigen Beobachtungsphase sind in $\mathrm{ng} / \mathrm{ml}$ angegeben. In der Kontrollgruppe lagen die Konzentrationen zwischen 14,6 und $19,7 \mathrm{ng} / \mathrm{ml}$. In der FFS-Gruppe kam es zu einem signifikanten Anstieg bis auf $160 \mathrm{ng} / \mathrm{ml}$. Bei der NaT-Pankreatitis ist der 40-min-Wert mit $452 \mathrm{ng} / \mathrm{ml}$ signifikant erhöht. Er steigt innerhalb von $2 \mathrm{~h}$ noch leicht auf $477 \mathrm{ng} / \mathrm{ml}$ an, um dann auf $270 \mathrm{ng} / \mathrm{ml}$ abzufallen und auf diesem Niveau zu verbleiben

Ascites

(experimentelle Pankreatitis)

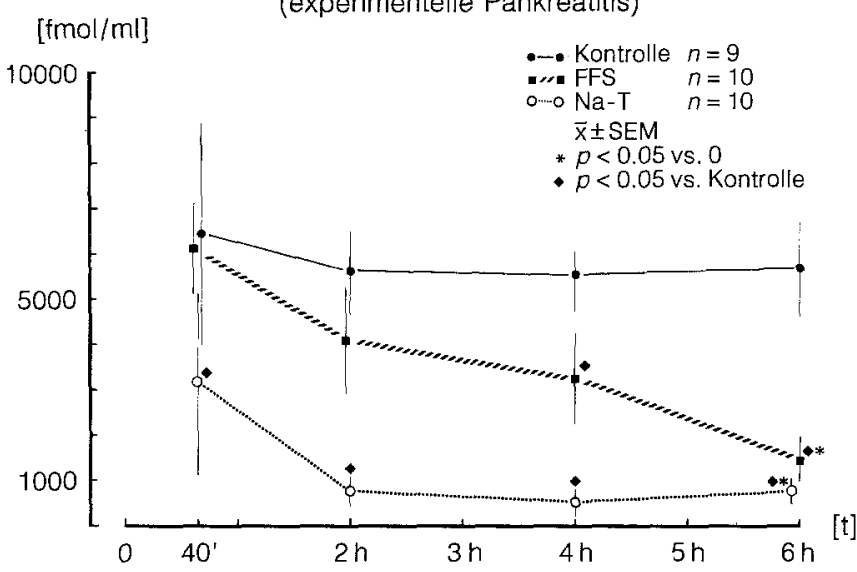

Abb. 4. Dargestellt sind die Mittelwerte ( \pm SEM) der Gesamtkininogenkonzentrationen in der Kontrollgruppe und in den beiden Pankreatitisgruppen (FFS und NaT) im Peritonealexsudat. Die Werte sind in $\mathrm{fmol} / \mathrm{ml}$ angegeben. Die Gesamtkininogenkonzentration änderte sich in der Kontrollgruppe nicht. In den Pankreatitisgruppen fand sich ein kontinuierlicher Abfall. In der FFS-Gruppe war der 6-h-Wert, in der NaT-Gruppe bereits der 40-min-Wert signifikant gegenüber der Kontrollgruppe erniedrigt

scher Lymphe während der Versuche annähernd gleich hoch, sie lag bei Versuchsbeginn knapp über $2 \mathrm{ng} / \mathrm{ml}$. In beiden Pankreatitisgruppen kam es zu einem Anstieg der Kallikreinkonzentration in Serum, Lymphe und Aszites (Abb. 5). Bei der FFS-Pankreatitis stiegen die Konzentrationen von Gewebekallikrein im Peritonealexsudat auf $160( \pm 51) \mathrm{ng} / \mathrm{ml}$ nach $6 \mathrm{~h}$. In der NaT-Gruppe zeigte sich ein rascher Anstieg von Gewebekallikrein in Plasma, peripankreatischer Lymphe und Peritonealexsudat (Abb. 3) schon innerhalb von $40 \mathrm{~min}$; die Konzentrationen stiegen dann weiter an bis Versuchsende ( $p<0,05$ vs. Kontrolle, vs. basal).

Die Gesamtkininogenkonzentration war in der Kontrollgruppe und in den Pankreatitisgruppen in der Lymphe niedriger als im Plasma. In allen 3 Gruppen kam es im Plasma und in der Kontrollgruppe und der $\mathrm{NaT}$ Gruppe auch in der Lymphe zu einem leichten, nicht signifikanten Abfall der Gesamtkininogenkonzentrationen; in der Lymphe der FFS-Gruppe fielen die Kininogenkonzentrationen über $6 \mathrm{~h}$ signifikant $\mathrm{ab}$ gegenüber dem Ausgangswert von $5457( \pm 933) \mathrm{fmol} / \mathrm{ml}$ und den Werten in der Kontrollgruppe. Im Peritonealexsudat wurde in beiden Pankreatitisgruppen ein signifikanter Abfall der Gesamtkininogenkonzentration über die gesamte Zeit beobachtet (Abb. 4), wobei der zeitliche Verlauf in den beiden Modellen unterschiedlich war. Bei der FFS-Gruppe war im Peritonealexsudat ein kontinuierlicher Abfall mit den niedrigsten Werten nach $6 \mathrm{~h}$ zu beobachten, während im NaT-Modell die Konzentration schon nach 40 min signifikant gegenüber der Kontrollgruppe vermindert war (Abb. 4).

\section{Diskussion}

Ein Ziel der Untersuchungen war es, die bei Pankreatitis initial im Pankreas und peripankreatisch stattfindende Freisetzung von Enzymen und die Aktivierung von Mediatorsystemen nachzuweisen. Das Pankreas wurde dazu in situ isoliert. Das Modell wurde an anderer Stelle ausführlich dargestellt [24], obwohl durch die Präparation am Intestinum die UUbertragbarkeit der gemessenen Werte auf andere Pankreatitismodelle beeinträchtigt ist.

Die klinische Diagnose der akuten Pankreatitis wird durch den Nachweis erhöhter Enzymaktivitäten gestützt. In beiden Pankreatitismodellen wurden erhöhte Lipaseund Phospholipase-A-Aktivitäten gemessen. Die Anstiege bewegen sich dabei in dem auch von anderen Gruppen beschriebenen Bereich. Während die Lipaseaktivität im Serum um das 3- bis 10 fache $[11,12]$ und in der Lymphe etwa um das 10fache [13] ansteigt, sind im Peritonealexsudat Anstiege bis zum hundertfachen des Ausgangswertes nachzuweisen [11-13]. Die Phospholipaseaktivität steigt bei der experimentellen Pankreatitis nur im Peritonealexsudat innerhalb der ersten $5 \mathrm{~h}$ meßbar an $[2,10$, 16]. In beiden Pankreatitismodellen läßt sich somit eine Freisetzung von Pankreasenzymen als biochemisches Zeichen der akuten Pankreatitis nachweisen.

Eine Aktivierung des Plasma-Kallikrein-Systems im Blut konnten wir in dem Modell der biliären Pankreatitis nicht nachweisen. Auch Ruud et al. [15] fanden bei der NaT-Pankreatitis innerhalb der ersten $5 \mathrm{~h}$ keine Aktivierung des Plasma-Kallikrein-Systems im Blut, sondern nur im Aszites.

Der Abfall des Plasmaprokallikreins im FFS-Modell läßt sich durch eine direkte Aktivierung an der durch die Ölsäure geschädigten Gefäßwand erklären [23].

In beiden Pankreatitismodellen waren ein Anstieg der Gewebekallikreinkonzentration und ein Abfall der Gesamtkininogenkonzentration in allen Kompartimenten 
zu beobachten. Die vermehrte Gewebekallikreinfreisetzung dürfte in Folge eines fehlenden effektiven Inhibitorpotentials zu einem vermehrten Kininogenabbau und damit verbunden zu einer beträchtlichen Kininfreisetzung führen. Der gemessene Abfall des Kininogens um etwa $50 \%$ im Peritonealexsudat ist deshalb bemerkenswert, da schon niedrige Kininogenkonzentrationen ausreichen, um starke lokale Wirkungen auszulösen. Auch von anderen Gruppen wurden Hinweise für eine Aktivierung des Gewebekallikrein-Kinin-Systems bei der akuten Pankreatitis beobachtet [17]. Kortmann [11] fand bei der NaT-Pankreatitis am Schwein im Plasma eine Verdoppelung der Gewebekallikrein und einen Abfall der Kininogenkonzentration auf ein Drittel des Ausgangswerts. Im Aszites stieg die Kallikreinkonzentration sogar um das 10- bis 100fache an, bezogen auf den Ausgangswert im Plasma, während die Kininogenkonzentration unter die Nachweisgrenze fiel [11]. In der vorliegenden Studie ist die Erhöhung der Gewebekallikreinkonzentration im Aszites wesentlich stärker ausgeprägt als in der Pankreaslymphe und hier stärker als im Plasma.

Durch die Messung von Gewebekallikrein und Kininogen im venösen Pfortaderblut, peripankreatischer Lymphe und im Peritonealexsudat gibt es starke Hinweise für eine Gewebekallikrein-Kinin-Aktivierung im Pankreas bei der experimentellen biliären Pankreatitis.

Durch die kontinuierliche Volumensubstitution, die stabile hämodynamische Bedingungen gewährleistete, konnten in unseren Versuchen Einflüsse der Hämodynamik etwa durch den hypovolämischen Schock auf biochemische Parameter minimiert werden. Eine Aktivierung des Kallikrein-Kinin-Systems ist somit wahrscheinlich durch die Pankreatitis bedingt. Pathophysiologisch dürfte der Aktivierung des Gewebekallikrein-Kinin-Systems eine große Bedeutung zukommen. Sie kann die ausgeprägten lokalen Veränderungen im Bauchraum, die Permeabilitätsstörung mit Flüssigkeitsverlust aus dem intravasalen Raum, mit Hypovolämie und Ödemen sowie die starken Schmerzen im Bauchraum bei akuter Pankreatitis erklären.

Bei der Pankreatitis wurden von Papp et al. [13] in Serum und Lymphe nur ein leichter Anstieg der Lipase beschrieben, das Verhältnis der Aktivitäten in den beiden Flüssigkeiten blieb dabei unverändert. Die Phospholipaseaktivität war unter Kontrollbedingungen in Lymphe und Serum etwa gleich. Bei der Pankreatitis stieg die Aktivität der PLA in der peripankreatischen Lymphe nicht an. Die Veränderungen der Konzentration von Komponenten des Kallikrein-Kinin-Systems war in der peripankreatischen Lymphe quantitativ stärker ausgeprägt als im Blut. Die relativ geringen Anstiege der Enzymaktivitäten in der Pankreaslymphe während der Pankreatitis lassen sich durch eine Verlegung intrapankreatischer Lymphbahnen erklären; bei der Pankreatitis kommt es in den geschädigten Regionen zum Austritt von Erythrozyten und Zelldetritus in die Lymphbahnen [1]. Die geringen Konzentrationsänderungen von Lipase und Phospholipase sowie von Kallikrein und Kininogen in der peripankreatischen Lymphe können in unseren Modellen der akuten Pankreatitis keinen wesentlichen Anteil an den systematischen Wirkungen haben.
Experimentell und klinisch werden im Peritonealexsudat bei der Pankreatitis um ein Vielfaches höhere Enzymspiegel als in Lymphe oder peripherem Blut gefunden [12, 20]. Auch vasoaktive Substanzen wie Kinine und Prostanoide wurden in hohen Konzentrationen nachgewiesen $[7,8,11,15,21,22]$.

Die Freisetzung in das Peritonealexsudat beginnt schon frühzeitig. Takada fand die höchsten Aktivitäten von Lipase, Amylase und Trypsin im Peritonealexsudat schon nach $1 \mathrm{~h}$; danach fielen die Aktivitäten ab [20]. Tierexperimentell konnte gezeigt werden, daß der frühzeitig gewonnene pankreatogene Aszites, wenn er gesunden Hunden i.v. verabreicht wird, eine hypotensive Wirkung hat, die im $16 \mathrm{~h}$ später gewonnenen Aszites nicht mehr nachweisbar ist. Der auch in unseren Versuchen gezeigte frühe Nachweis hoher Enzymkonzentration und die Veränderungen der Konzentration von Gewebekallikrein und Kininogen im Peritonealexsudat sprechen für eine initial lokale Aktivierung und Freisetzung bei der Pankreatitis. Durch die konsequente Ableitung des Aszites aus dem Bauchraum wurde die Resorption in den systemischen Kreislauf minimiert. Dies kann die geringen Anstiege von Lipase und PLA im Blut erklären. Im Gegensatz dazu konnten Mayer et al. [12] am Hund durch die Separation von Aszites die Enzymspiegel im Blut nicht vermindern. In seinem Modell erfolgte die Messung aber nur an dem überwiegend retroperitoneal gelegenen Pankreaskorpus und Pankreasschwanz mit Drainage durch retroperitoneale Lymphbahnen, so da $B$ die Abgabe von Enzymen und Mediatoren in den freien Bauchraum sicher geringer war als in unserem Modell.

Es konnte gezeigt werden, daß es in beiden Pankreatitismodellen zu einer frühzeitigen Freisetzung der Lipase in venöses Pankreasblut, Pankreaslymphe und einer Aktivierung der Phospholipase im Peritonealexsudat kommt. Der Anstieg der Gewebekallikreinkonzentration und der Abfall des Gesamtkininogens bei nahezu unverändertem Gesamteiweiß in beiden Pankreatitismodellen sind ein Hinweis auf eine Aktivierung des Gewebekallikrein-Kinin-Systems. Die Veränderungen sind dabei jeweils im Peritonealexsudat am ausgeprägtesten. Die akute Pankreatitis ist initial ein lokales Geschehen im Pankreas und seiner Umgebung. Die Freisetzung und Aktivierung der Enzyme und die Änderung der Konzentration von Kallikrein und Kininogen in den Kompartimenten venöses Pankreasblut, Lymphe und Peritonealexsudat ist unterschiedlich. Im Peritonealexsudat lassen sich um ein Vielfaches (bis zu 50fach) höhere Konzentrationen nachweisen als in Lymphe oder venösem Pankreasserum, was für die ,Vermittlung" systematischer Wirkungen berücksichtigt werden muß.

\section{Literatur}

1. Anderson MC, Schiller WR (1968) Microcirculatory dynamics in the normal and inflamed pancreas. Am J Surg 115:118

2. Ascherl R, Müller F, Kurz M, Werner E, Wimmer B, Blümel G (1987) Phospholipase A (PLA) und akute hämorrhagischnekrotisierende Pankreatitis - Experimentelle Untersuchungen. Langenbecks Arch Chir [Suppl] 349 
3. Balldin $G(1980)$ On protease-antiportease imbalance with special reference to the protective role of protease inhibitors in acute pancreatitis. Akademisk Achandling, Malmö

4. Bradley EL, Hall JR, Lutz J, Hamner L, Latthouf O (1983) Hemodynamic consequences of severe pancreatitis. Ann Surg 198:130

5. Cobo LC, Abraham E, Bland RB (1984) Sequential hemodynamic and oxygen transport abnormalities in patients with acute pancreatitis. Surgery 95:324

6. Creutzfeld W, Lankisch PG (1985) Acute pancreatitis: etiology and pathogenesis. In: Berk JE (ed) Gastroenterology VI, Saunders, Philadephia London Toronto New Mexico Rio de Janeiro Sydney Tokyo

7. Farias LR, Frey CF, Holcroft JW, Gunther R (1985) Effect of prostaglandin blockers on ascites fluid in pancreatitis. Surgery 98:571

8. Galzer G, Gilliland EL, Aldridge MA (1987) The role of prostaglandins in acute pancreatitis. Surg Annu 19:175

9. Hoffmann GE, Schmidt D, Bastian B (1985) Bestimmung der Phospholipase A im Serum bei akuter Pankreatitis. J Clin Chem Clin Biochem 23:582-583

10. Hölbling N, El-Kalak H, Georgopoulos A et al. (1985) Phospholipase $\mathrm{A} 1$ and $\mathrm{A} 2$ in experimental acute pancreatitis in rats. Res Exp Med 185:131

11. Kortmann H (1984) Limitierte und unspezifische Proteolyse beim pankreatogenen Schock. Habitationsschrift, Universität München

12. Mayer AD, Airey M, Hodgson J, McMahon MJ (1985) Enzyme transfer from pancreas to plasma during acute pancreatitis. The contribution of ascitis fluid and lymphatic drainage of the pancreas. Gut 26:876

13. Papp N, Nemeth EP, Horvath EJ (1971) Pancreaticduodenal lymph flow and lipase activity in acute experimental pancreatitis. Lymphology 4:48

14. Pissas A (1984) Anatomoclinical and anatomosurgical essay on the lymphatic circulation of the pancreas. Anat Clin $6: 255$
15. Ruud TE, Aasen AO, Kierulf P, Stadaas J, Aune S (1985) Studies in the plasma during experimental acute pancreatitis in pigs. Scand J Gastroenterol 20:877

16. Schröder T, Lempinen M, Nordling S, Kinmenen PKJ (1981) Chlorpromazine treatment of experimental acute fulminant pancreatitis in pigs. Eur Surg Res 13:143

17. Seung WP, Feldman BF (1985) Early phase components of the kallikrein kinin system in hemorrhagic ascitic fluid and plasma in the rat with induced acute pancreatitis. Am J Vet Res 46:1961

18. Siebeck M, Fink E, Weipert J, Jochum M, Fritz H, Spannagel M, Kroworsch P, Shimamoto K, Schweiberer L (im Druck) Inhibition of plasma kallikrein with aprotinin in porcine endotoxin shock. J Trauma

19. Steer ML, Meldolesi J (1984) Experimental acute pancreatitis: Relevance of models to clinical disease. In: Gyr KE, Singer MV, Sarles H (eds) Pancreatitis - Concepts and classification. Excepta Medica, Amsterdam New York Oxford

20. Takada T, Apper HE et al. (1976) Vascularpermeability induced by pancreatic exudate-formed during acute pancreatitis in dogs. Surg Gynecol Obstet 143:779

21. Traverso LW, Pullos TG, Frey CF (1983) Hemodynamic characterization of porcine hemorrhagic pancreatitis ascites fluid. $J$ Surg Res 34:254

22. Vollmar B, Waldner H, Schmand J, Conzen PF, Goetz AE, Habazettl H, Schweiberer L, Brendel W (1989) Release of arachidonic acid metabolites during acute pancreatitis in pigs. Scand J Gastroenterol 24:1253-1264

23. Vollmar B, Waldner H, Schmand J, Conzen PF, Goetz AE, Habazettl H, Schweiberer L, Brendel W (1991) Oleic acid induced pancreatitis in pigs. J Surg Res 50:1-9

24. Waldner H, Schmand J, Vollmar B, Goetz A, Conzen P, Schweiberer L, Brendel W (1990) Die Pankreasdurchblutung bei der experimentellen biliären Pankreatitis. Langenbecks Arch Chir 375:112-118

25. Weber H (1965) Mikromethode zur Bestimmung der Pankreaslipase im Serum. Dtsch Med Wochensch 26:1170 